\title{
j-NICHE: Prototype detectors of a non-imaging Cherenkov array at the Telescope Array site
}

\author{
Douglas R Bergman* \\ The University of Utah \\ E-mail: bergman@physics.utah.edu \\ Yoshiki Tsunesada \\ Osaka City University \\ E-mail: ytesci.osaka-cu.ac.jp

\section{John F Krizmanic} \\ University of Maryland, Baltimore County \\ E-mail: jkrizman@umbc.edu

\section{Yugo Omura} \\ Osaka City University \\ E-mail: omura@cosmicray-ocu.jp
}

The Non-Imaging CHErenkov Array (NICHE) is a low energy extension to Telescope Array and TALE using an array of closely spaced ( $\sim 200 \mathrm{~m})$ light collectors covering an area of $\sim 2$ square $\mathrm{km}$. It is being deployed in the field of view of TALE and will overlap it in the energy range above $4 \mathrm{PeV}$. Cosmic ray air showers with energies $1-100 \mathrm{PeV}$ will be reconstructed using both the Cherenkov light Lateral Distribution and the Cherenkov time Width Lateral Distribution. These two methods will allow shower energy and $X_{\max }$ to be determined. A prototype of the array, called j-NICHE, is currently being built and deployed. The design and plans for the full array are presented along with a plan to deploy the first 15 counters to get a true Cherenkov hybrid air shower measurement. Performance of the detectors, calibration details and preliminary data are presented.

35th International Cosmic Ray Conference - ICRC2017

10-20 July, 2017

Bexco, Busan, Korea

* Speaker. 


\section{Scientific Motivation}

Measurement of the changing nuclear composition of high-energy Cosmic Rays (CR) at energies around the Knee, $\gtrsim 1 \mathrm{PeV}$, provides a unique tool for understanding the evolution of the high-energy end of the galactic CR spectrum while providing a firm foundation for understanding the composition and spectrum of extragalactic ultra high-energy CRs. However, the current understanding is muddled due to uncertainties inherent to the measurement techniques and/or dependence on hadronic Monte Carlo simulation models required to interpret the data[1]. This ambiguity makes it difficult to confirm the widely adopted hypothesis that the Knee is the result of a Peters cycle[2] due to rigidity-dependent cutoffs of the various galactic CR nuclear components.

One way to minimize this state of uncertainty is to perform a coordinated set of experiments to observe the composition of CRs over many orders-of-magnitude, using techniques that are directly sensitive to composition-dependent observables. One such sensitive observable for measuring CR composition in air showers is the depth of shower maximum, $X_{\max }$, which is measurable in Fluorescence Telescopes (FTs), Imaging Air-Cherenkov Telescopes (IACTs), and non-imaging Cherenkov Detectors (CDs). Historically, non-imaging CDs and FTs have not been used together to simultaneously measure air showers. However, with the newly demonstrated ability of FTs to work as IACTs when pointed at larger elevation angles[3], it has become possible to combine FTs (as IACTs) and CDs in a hybrid array. In addition, by using both the lateral distribution of Cherenkov photons and the time-domain structure of their arrival times, the spacing of Cherenkov counters can be sufficiently increased and lead to a large enough aperture to overlap with lower-energy FTs (qua FTs) such as TALE. Thus, the measurement of the air-Cherenkov signal on the ground, calibrated with air fluorescence and imaging air-Cherenkov measurements, offers a methodology to provide an accurate measurement of the nuclear composition evolution over a large energy range. NICHE will use an array of easily deployable, non-imaging Cherenkov detectors with a variety of spacings to measure the amplitude and time-spread of the air shower Cherenkov signal to extract CR nuclear composition measurements and to cross-calibrate the Cherenkov energy and composition measurements with that from TA/TALE fluorescence and surface detectors[4].

\section{The Non-imaging Cherenkov Technique}

Energetic electrons generated in an extended air shower (EAS) produce Cherenkov radiation if they move faster than the speed-of-light in the local medium. The index of refraction in the atmosphere increases with depth, yielding Cherenkov cones from altitudes 8-20 km to overlap in a ring of radius 120-140 $\mathrm{m}$ at the ground. Since the interior of the ring is filled by the portion of the air shower at lowest altitudes, showers that develop deeper in the atmosphere will have a larger interior-to-ring ratio. Electron transverse momentum smears the Cherenkov ring on the ground, but the inside-to-outside ratio remains composition, i.e. $X_{\max }$, dependent.

At a given point on the ground, a counter will observe two components of Cherenkov light: one from the bulk of the shower, where some fraction of the transverse electrons are pointing their Cherenkov cones at the counter; and another part due to the smaller portion of the shower where the shower core Cherenkov cone intercepts the counter. Photons in the former component arrive over a long time span because the measurement samples a large portion of the developing air shower, 
while the latter component is narrow in time. When there is no dominant core component observed in a counter, the FWHM can be quite wide. Thus the FWHM in time provides a measure of shower development: deeper showers will have more Cherenkov light coming late due to sampling the air shower over a long path length.

A number of experiments have employed the non-imaging Cherenkov technique (AIROBICC, BLANCA, CACTI, and Tunka) using the Cherenkov Light Distribution (CLD) to measure the CR spectrum, while two experiments (Tunka \& BASJE) have employed the Cherenkov Time Width Lateral distribution technique (references are listed in [5]). The innovation of NICHE is to combine these two techniques to construct an array of sufficiently large area to have significant overlap with TALE air-Cherenkov measurements for $E \gtrsim 1 \mathrm{PeV}$ and TA/TALE air fluorescence measurements for $E \gtrsim 100 \mathrm{PeV}$, leading to a cross-calibration of the FT, IACT and non-imaging CD measurements.

\section{3. j-NICHE}

A "Kakenhi" Grant by Japan Society for the Promotion of Science (JSPS) was approved in 2014 for four years. It will provide for a prototype array of 13 CDs to be called j-NICHE. Funds from the first year of this grant were used to purchase photomultiplier tubes; specify and procure an FADC data acquisition system from Brains, Inc. in Japan; purchase batteries and solar panels; and design and procure Winston cones (milled in the Dept. of Physics and Astronomy at the University of Utah). Funds from the second year were used for the design and manufacture of detector enclosure (at the University of Utah); design and construction of a control box for controlling the shutter and PMT high voltage; and for testing of the Brains DAQ system. Funds from the third year were used for the assembly of the counters in Delta, Utah and for the deployment of four detectors into the field.

The planned layout of the full NICHE array and the j-NICHE prototype array are shown in Figure 1. One of the j-NICHE sites will have two CDs to check coincidences. The locations of the four deployed counters is also shown in Figure 1. The NICHE array is a small-spacing array with a small area since it is intended to overlap in energy with the TALE FD acting as an IACT[6]. The j-NICHE array will instrument a subset of the NICHE sites as shown.

The detector enclosures have been constructed at the University of Utah. The enclosure has a porthole on the top, under which a rotating platter opens and closes a shutter. The enclosure is attached to a stand which also contains a solar panels and masts for a communications antenna and a GPS antenna. A picture of one enclosure/stand is shown on the left in Figure 2. In side the enclosure are two batteries, a Winston cone, a photomultiplier tube, the DAQ/FADC box (from Brains Inc.), a control box, and a charge controller. The interior of the detector enclosure is shown on the right in Figure 2.

\section{The First Data from j-NICHE}

j-NICHE was first operated on 22 March 2017 with one detector (named Newton) functioning. The first waveform of a Cherenkov signal is shown in Figure 3. Two CDs (Newton, and another named Curie) were run on the nights of 20 and 21 April 2017 and 26 May 2017. A total of 15 hours 

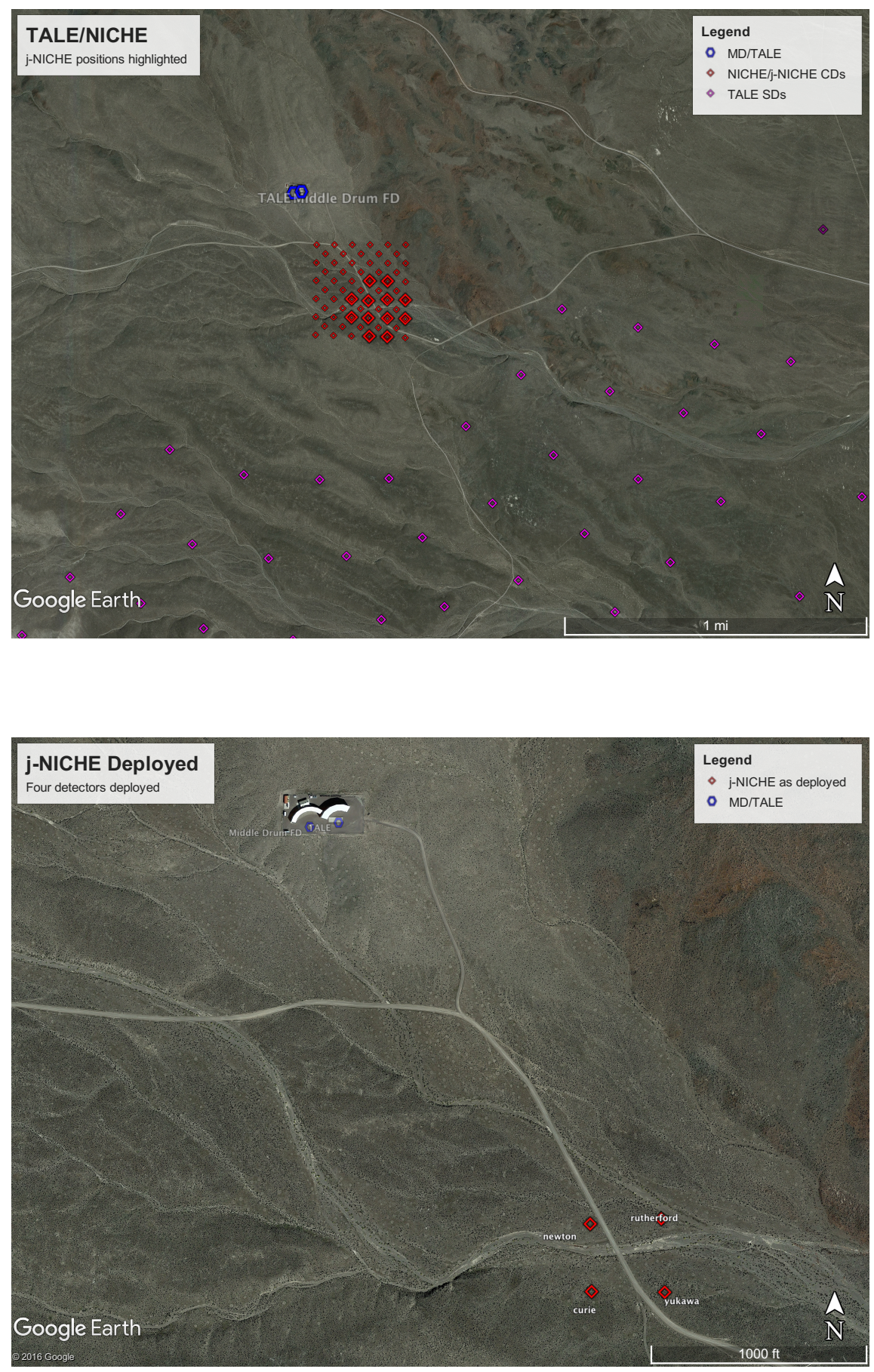

Figure 1: In the upper panel, the planned layout of NICHE and j-NICHE with respect to the MD/TALE FDs and TALE SDs. The small red diamonds denote the planned locations of the full NICHE array with 61 counters with $71 \mathrm{~m}$ separation. The large red diamonds denote the planned locations for the prototype $\mathrm{j}$-NICHE array. In the lower panel, the actual positions of the four deployed j-NICHE counters with respect to the MD/TALE FDs 

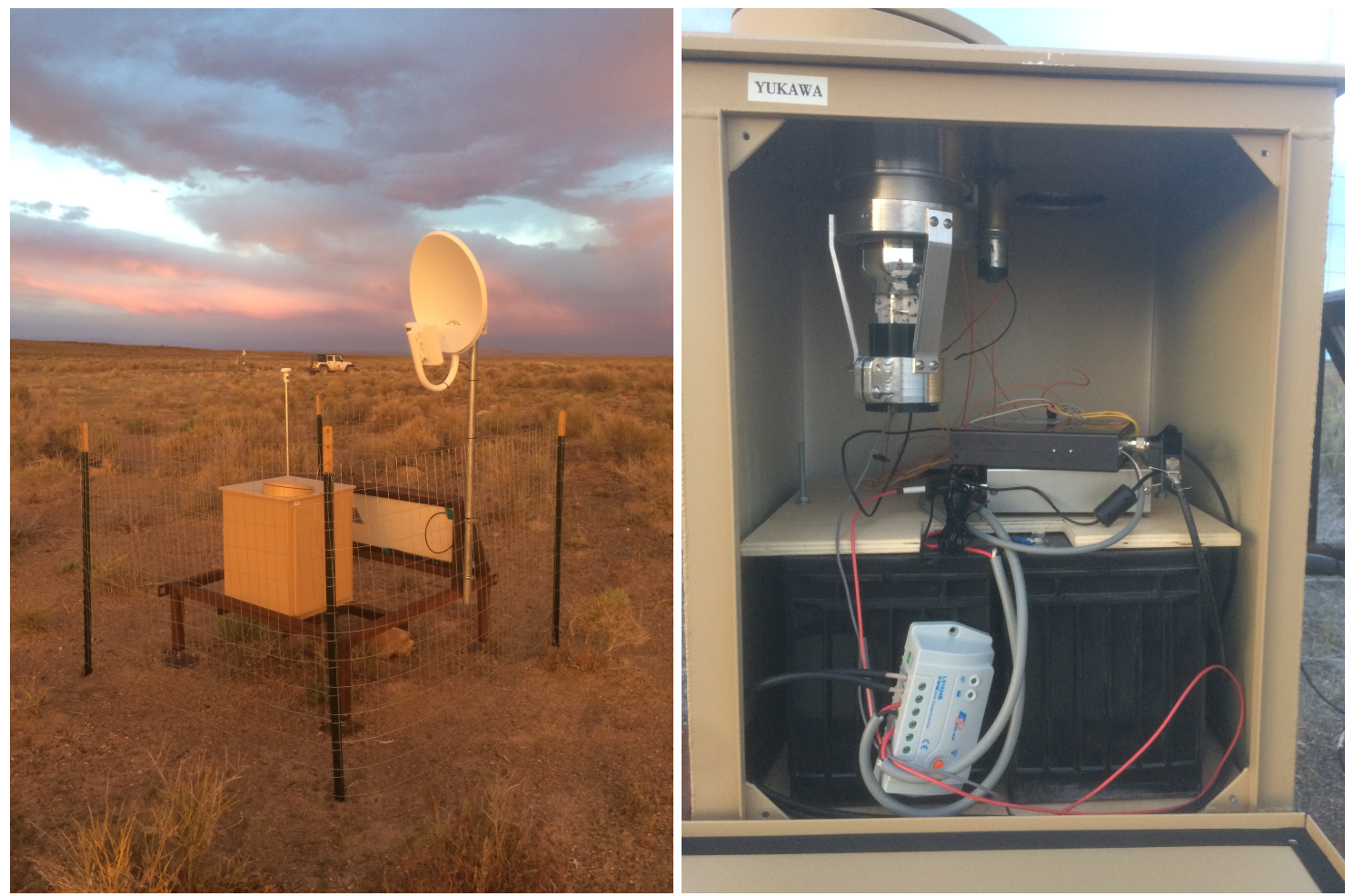

Figure 2: On the left, a deployed detector in the field, showing detector enclosure, stand, solar panel, communications antenna and GPS antenna. On the right, the interior of a detector enclosure, showing photomultiplier tube, DAQ/FADC box, control box, two batteries, and charge controller.

of data was taken with 24 coincidences between Newton and Curie found. The time differences in the coincidences span a range of $\pm 180 \mathrm{~ns}$, which corresponds to zenith angles up to $30^{\circ}$ given the $100 \mathrm{~m}$ separation. The largest signal in these NICHE coincidences also coincides with a TALE FD event as shown in Figure 4.

\section{References}

[1] K.-H. Kampert, M. Unger, Measurements of the cosmic ray composition with air shower experiments, Astropart. Phys. 35 (2012) 660.

[2] B. Peters, Primary energy spectrum and air showers, Proceedings of the 6th ICRC (Moscow) 3 (1960) 157.

[3] T. Abu-Zayyad, Cosmic Rays Energy Spectrum observed by the TALE detector using Cerenkov light, PoS(ICRC2015)422.

[4] J. Krizmanic, D. Bergman, P. Sokolsky, The Non-Imaging CHErenkov Array (NICHE): A TA/TALE Extension to Measure the Flux and Composition of Very-High Energy Cosmic Rays, Proceedings of the 33rd ICRC (Rio de Janeiro), (2013) 0365.

[5] D. Bergman, J. Krizmanic, NICHE: The non-imaging Cherenkov array, AIP Conf. Proceed. 1516 (2013) 282. 
wf 2017-03-22 03:18:45.109585350

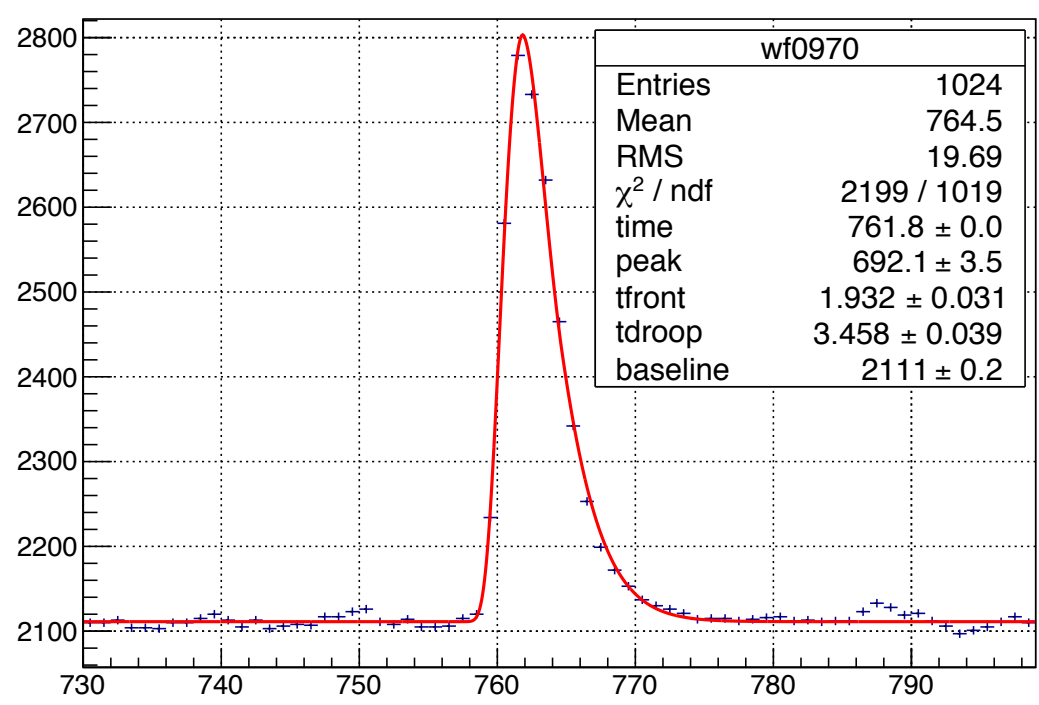

Figure 3: The first waveform from an air shower observed with $\mathrm{j}$-NICHE (from Newton). The $x$-axis is in units of FADC samples (5 ns), the $y$-axis is in units of FADC counts, with no signal corresponding to a mid-scale value of around 2048. The fit of the waveform pulse shape is from a form used by the Tunka Collaboration[7].

[6] D. R. Bergman, J. F. Krizmanic, Y. Tsunesada, The NICHE Array: Status and Plans, Proceedings of the 34th ICRC (The Hague), PoS(ICRC2015)635.

[7] E.E. Korosteleval, L.A. Kuzmichev, V.V. Prosin, A.V. Zablotsky, The New Method of EAS Parameters Reconstruction Using the FWHM of Cherenkov Light Pulses, Proceedings of the 31st ICRC (Łódź), 0492. 

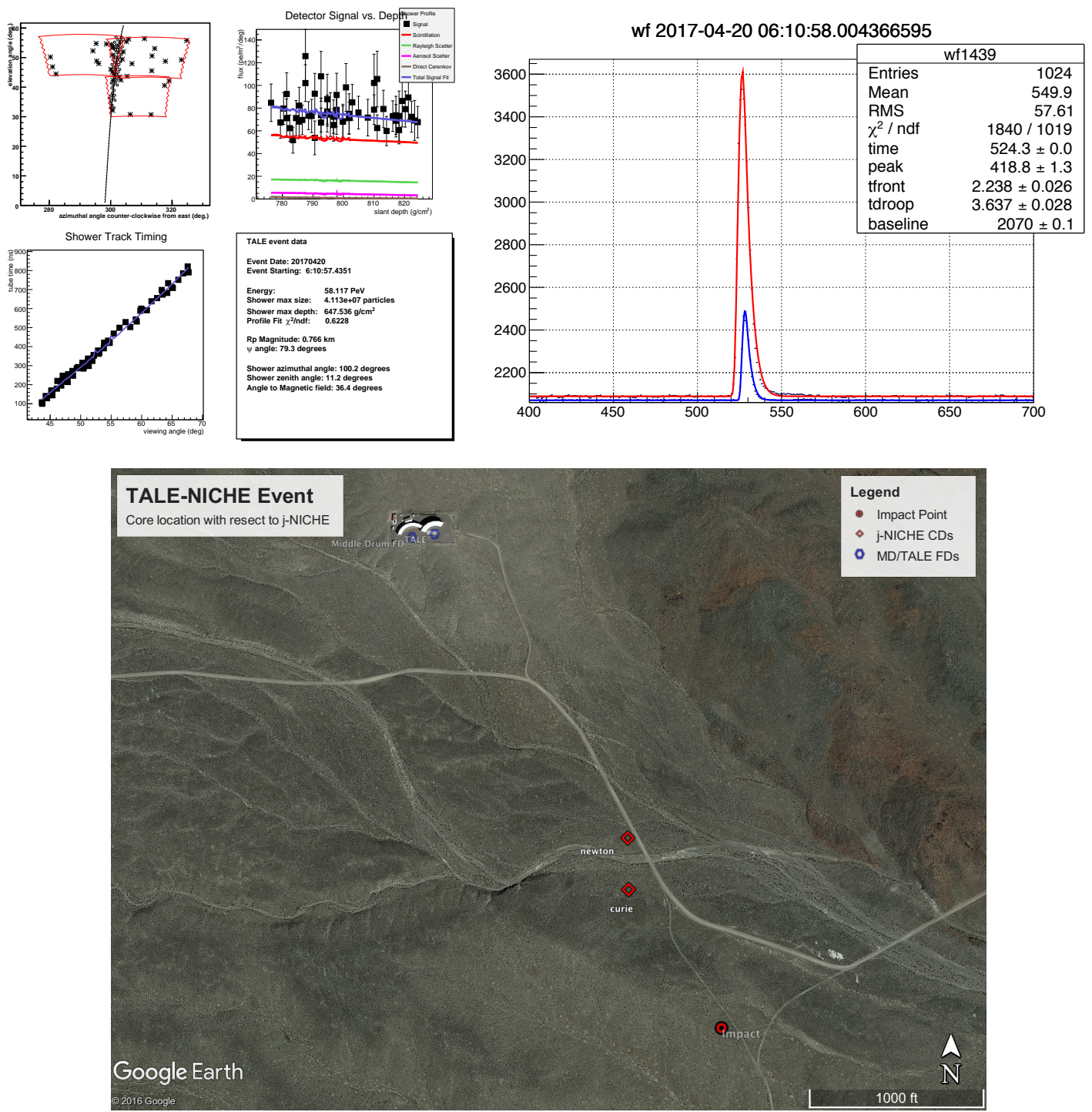

Figure 4: A TALE/j-NICHE coincident event. The upper-left set of panes shows the TALE FD reconstruction which includes a shower-detector-plane fit and a reconstruction of the geometry. From this the impact point of the shower can be estimated and is drown on the lower panel with respect to the j-NICHE counters. The waveforms of the two j-NICHE counters are shown in the upper-right. The $x$-axis is in units of FADC samples $(5 \mathrm{~ns})$, the $y$-axis is in units of FADC counts, with no signal corresponding to a mid-scale value of around 2048. The TALE-reconstructed zenith angle is $11.2^{\circ}$ leading to nearly simultaneous signals in j-NICHE. 